\title{
Lamb-shift Polarized Ion Source at UTTAC
}

\author{
T. Moriguchi*, A. Ozawa, Y. Yamato, S. Suzuki, M. Amano, D. Kamioka \\ University of Tsukuba \\ E-mail: moriguchi@tac.tsukuba.ac.jp \\ D. Nagae, Y. Abe
}

RIKEN, Nishina Center

The Lamb-shift polarized ion source (PIS) was installed as one of the ion sources for the 12UD Pelletron tandem accelerator at the University of Tsukuba Tandem Accelerator Complex (UTTAC) in 1976. The PIS makes it possible to produce highly polarized negative proton and deuteron beams. However, the Great East Japan Earthquake on 11 March 2011 seriously damaged not only the accelerator but also the PIS. Following the disaster, it was decided that the PIS should be reconstructed and used as the ion source for the newly designed $6 \mathrm{MV}$ tandem accelerator. Main components including the duoplasmatron chamber and spin filter were reused, while other components including the accelerator tubes and some beam ducts were newly produced. After the reconstruction of the PIS, we attempted to generate polarized beams and measure the polarization. Recently, we attempted to produce unstable nuclei with the polarized proton beam for measurements of nuclear moments of unstable nuclei.

XVII International Workshop on Polarized Sources, Targets \& Polarimetry

16-20 October 2017

Kaist, South Korea

*Speaker. 


\section{Introduction}

A polarized ion beam is an effective tool for measurements of physical quantities such as analyzing power and nuclear magnetic moments. The Lamb-shift polarized ion source (PIS) was installed at the University of Tsukuba Tandem Accelerator Complex (UTTAC) in 1976 for experimental nuclear physics [1]. With the PIS, it is possible to produce highly polarized negative proton and deuteron beams. The PIS was used as one of three ion sources for the 12UD Pelletron tandem accelerator. However, the Great East Japan Earthquake on 11 March 2011 seriously damaged not only this accelerator but also the PIS. After the disaster, the decision was made to reconstruct the PIS along with the installation of the newly designed $6 \mathrm{MV}$ tandem accelerator. Before the disaster, the PIS was located at the 9th floor of the main building. In order to combine the PIS with the new accelerator, a new building for the PIS was constructed outside of the main building as shown in Fig. 11 (a) [2]. Since the main components of the PIS such as the duoplasmatron chamber, the spin filter, the argon gas cell, the electrostatic steerer, and the Wien filter were reusable, they were moved from the 9th floor of the main building to the new building, and then were reconstructed. The remaining components of the PIS were essentially replaced with new version. By March 2015, almost all of the reconstruction of the PIS had been completed, as shown in Fig. 1(b).

(a)

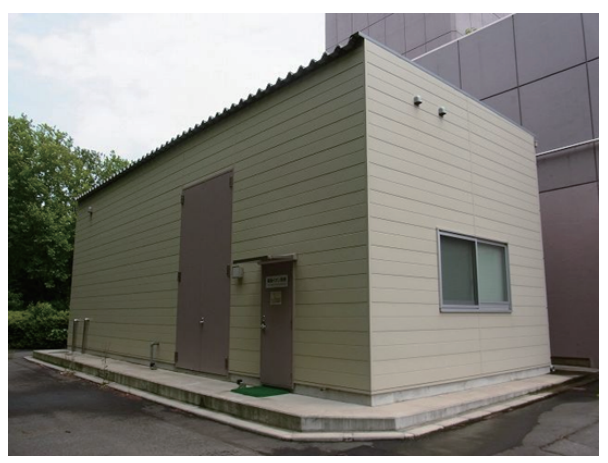

(b)

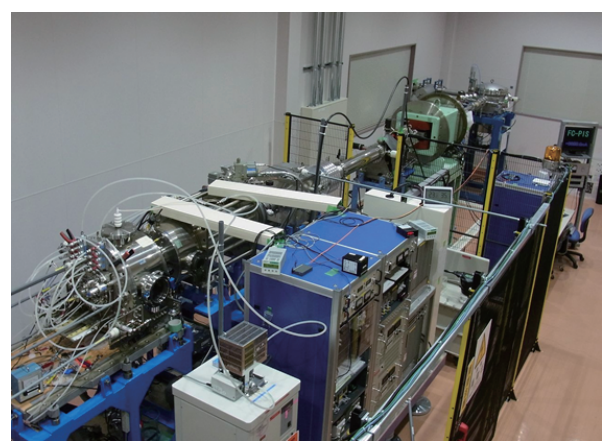

Figure 1: (a) The PIS building. (b) The PIS after the reconstruction.

\section{Lamb-shift polarized ion source}

Figure 2 shows a schematic diagram of the Lamb-shift polarized ion source at UTTAC. The Lamb shift refers to the energy difference $\left(4.4 \times 10^{-6} \mathrm{eV}\right)$ between the $2 S_{1 / 2}$ and $2 P_{1 / 2}$ states of hydrogen and deuterium, as shown in Fig. 3 The polarized proton beams are generated as follows. Positive hydrogen ions, which are extracted from the duoplasmatron, are selectively changed to hydrogen atoms at the $2 S_{1 / 2}$ state in cesium vapor, and these atoms are transmitted through the deflector since ions are eliminated by these deflection plates. The spin filter (SF) is of significance for the nuclear polarization [1]. The SF is designed to provide (1) a uniform magnetic field parallel to the beam axis, (2) a $1610 \mathrm{MHz}$ radio-frequency (RF) electric field parallel to the beam, and (3) a static electric field perpendicular to the beam. Through the proper adjustment of these SF parameters, hydrogen atoms in metastable states, which are indicated by dotted lines in Fig. 3 , 
are transmitted through the SF. This is based on the phenomenon referred to as the "three-level interaction" [3] [4]. This phenomenon results in the production of the nuclear polarization, because the nuclear spin magnetic quantum number $m_{\mathrm{I}}$ of these metastable atoms is either $+1 / 2$ (540 Gauss) or $-1 / 2$ (605 Gauss), regardless of the electron spin, as shown in Fig. 3 . Atoms in other states are quenched to the ground state immediately in the SF. In the argon gas cell, these metastable atoms are selectively ionized to negative hydrogen ions, whereas atoms in the ground state are not. Thus, polarized proton beams can be generated from the PIS. Polarized deuteron beams can also be generated through the same principles as the polarized proton beam.

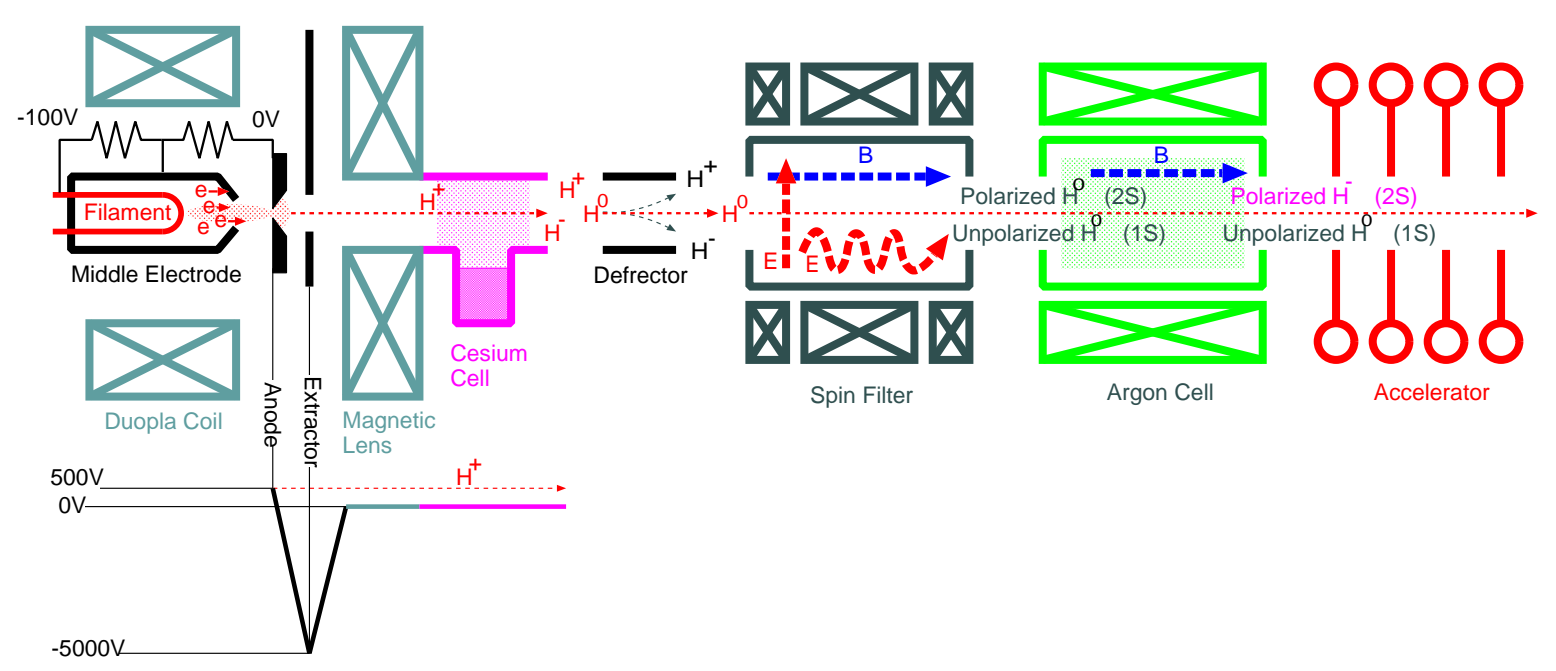

Figure 2: Schematic diagram of the Lamb-shift polarized ion source at UTTAC.
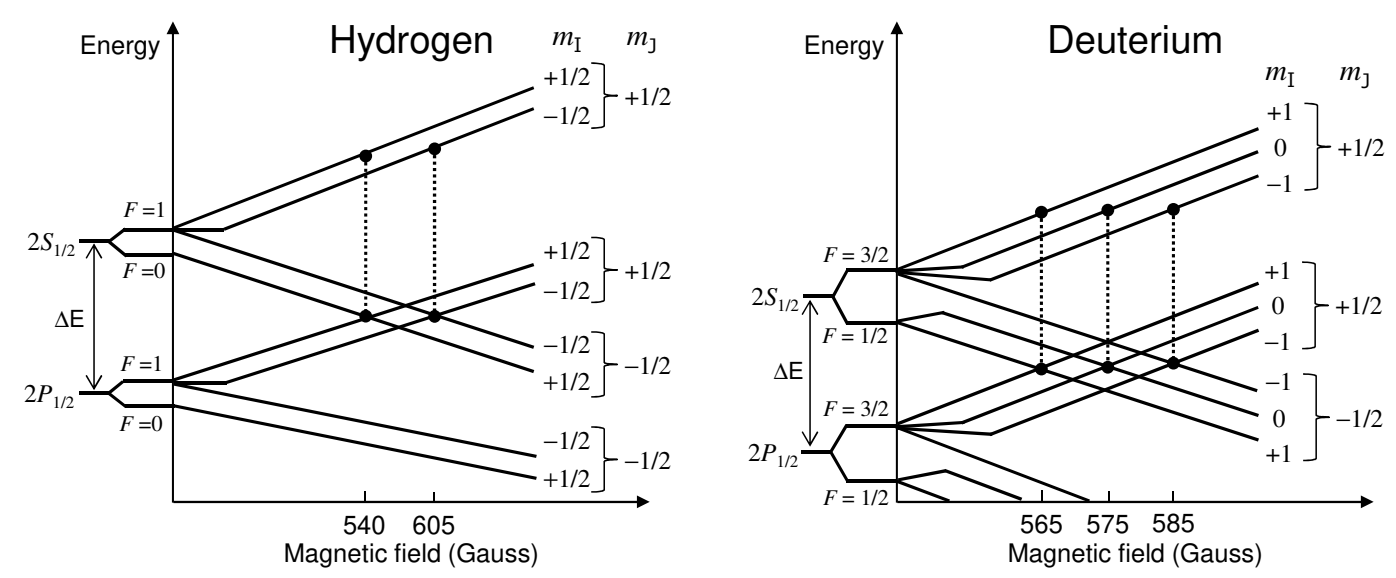

Figure 3: Hyperfine splitting of hydrogen (left panel) and deuterium (right panel) in an external magnetic field. $\Delta \mathrm{E}$ represents the Lamb shift. $m_{\mathrm{I}}$ and $m_{\mathrm{J}}$ are the nuclear spin magnetic quantum number and the electron spin magnetic quantum number, respectively. 


\section{Measurements of Polarization}

Following the reconstruction of the PIS, we studied the polarization of the polarized beams. The beam current was measured using a Faraday cup (FC-PIS) located just downstream of the PIS. Figure 4 shows the measured beam current as a function of the SF magnetic field. A negative current was measured because negatively charged protons or deuterons were extracted from the PIS. For the proton beam, we found two resonance peaks around 540 and $605 \mathrm{G}$, which correspond to $m_{\mathrm{I}}=+1 / 2$ and $-1 / 2$, respectively. We found three resonance peaks for the deuteron beam around 565,575 , and $585 \mathrm{G}$, which correspond to $m_{\mathrm{I}}=+1,0$, and -1 , respectively. From this measurement, we could estimate the polarization of the polarized beam. Realistically, the unpolarized beam is partly included as background in the polarized beam. The quenching beam, which can be produced by shifting the SF magnetic field from the resonant field, includes only the unpolarized part. Using the beam current of the polarized beam $I_{\mathrm{pol}}$ and the quenching beam $I_{\text {unpol }}$, the polarization $P$ can be described by $P=\frac{I_{\mathrm{pol}}-I_{\mathrm{unpol}}}{I_{\mathrm{pol}}}$. This is called the quenching method. $I_{\mathrm{pol}}$ and $I_{\text {unpol }}$ correspond to the peak current and the base current in Fig. 4 respectively. Using the beam current of the FC-PIS, we found that the polarization of the $m_{\mathrm{I}}=+1 / 2$ peak shown in Fig. 4 was about $80 \%$. This is consistent with the value of the PIS before the disaster [1].

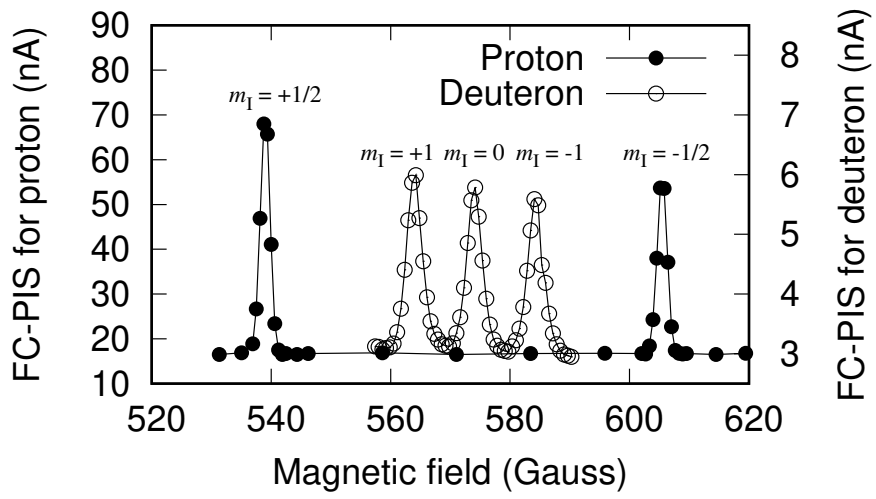

Figure 4: Beam current measured by the Faraday cup (FC-PIS) as a function of the magnetic field.

After the estimation of the polarization at the PIS, we measured that of a proton beam downstream of the $6 \mathrm{MV}$ tandem accelerator with a polarimeter using the $p-{ }^{4} \mathrm{He}$ elastic scattering reaction. Figure 5 (a) shows the schematic cross section of the polarimeter [5]. The cross section shown in Fig. [5 (a) is perpendicular to the spin quantization axis of the polarized beam. The inside of the polarimeter was filled with ${ }^{4} \mathrm{He}$ gas of approximately $0.1 \mathrm{MPa}$. Elastically scattered protons in the polarimeter traveled through the intervals of each vane and were detected by silicon detectors at both sides. In this measurement, the polarized proton beam from the PIS was accelerated to 12 $\mathrm{MeV}$ and transported to the experimental beam course. The spin quantization axis was set to be perpendicular to the horizontal plane and the direction of the spin (spin-up or spin-down) could be selected by reversing the direction of the SF magnetic field. The polarimeter was connected to the end of the beam course as shown in Fig. 5(b). Polarization $P$ is expressed as

$$
A \cdot P=\frac{N_{\mathrm{L}}-N_{\mathrm{R}}}{N_{\mathrm{L}}+N_{\mathrm{R}}}
$$


where $A$ is the analyzing power; $N_{\mathrm{L}}$ and $N_{\mathrm{R}}$ are the number of elastically scattered protons detected by the silicon detectors on the left and right sides of the polarimeter, respectively. In this case, $A$ is approximately equal to one [6]. Figure [6] shows the experimental results obtained from both silicon detectors. The main peaks shown in Fig. 6 correspond to the energy of the elastically scattered protons. In the case of spin-up (spin-down), the number of the protons scattered to the left (right) side is larger than that to the other side, as shown in Fig. 6. From this experimental result and using Eq. 3.1, we found that the polarization at the end of the beam line was approximately $44 \%$ for the spin-up case and $36 \%$ for the spin-down case. These polarizations are lower than the polarization of downstream of the PIS obtained using the quenching method, as mentioned above. One of the reasons for this depolarization may be the electron exchange between ions and a residual gas inside the beam duct in the accelerator. In the general case of the tandem accelerator, negatively charged ions from ion sources are accelerated towards the positively charged high voltage terminal. The electrons are then stripped for the second acceleration using positively charged ions. Our tandem accelerator has thin carbon foils and a canal filled with argon gas as the electron stripper, which can be selected depending on the experimental conditions. In this measurement, a thin carbon foil (approximately ten $\mu \mathrm{g} / \mathrm{cm}^{2}$ ) was used to suppress the depolarization. However, owing to possible defects in the gas valve or vacuum system, argon gas might have leaked slightly into the canal. This would increase the depolarization.

(a)

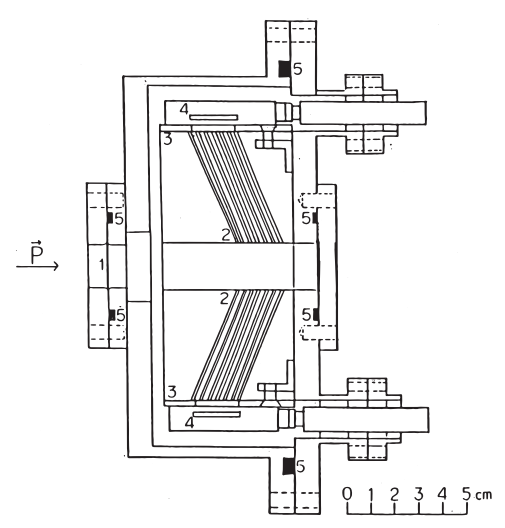

(b)

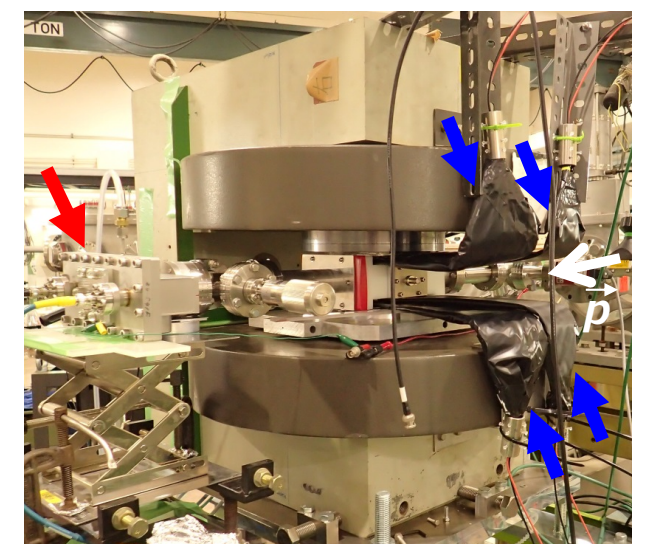

Figure 5: (a) Schematic cross section of the polarimeter with labels: 1. Harver foils, 2. vanes, 3. diaphragms, 4. silicon detectors, and 5. O-ring [5]. (b) Experimental setup at the end of the beam course. The white and red arrows indicate the direction of the polarized proton beam and the polarimeter, respectively. Blue arrows indicate plastic scintillation counters for detecting the $\beta$-ray emitted from the unstable nuclei, as explained in Sec. 4

\section{Production of Unstable Nuclei}

Recently, we attempted to produce unstable nuclei with a polarized proton beam from the PIS. This is a first step for measurements of the nuclear moments of unstable nuclei. The polarized proton at an energy of $12 \mathrm{MeV}$ was irradiated to a beam stopper of natural silicon $\left({ }^{28} \mathrm{Si}: 92.2 \%\right.$, 
(a)

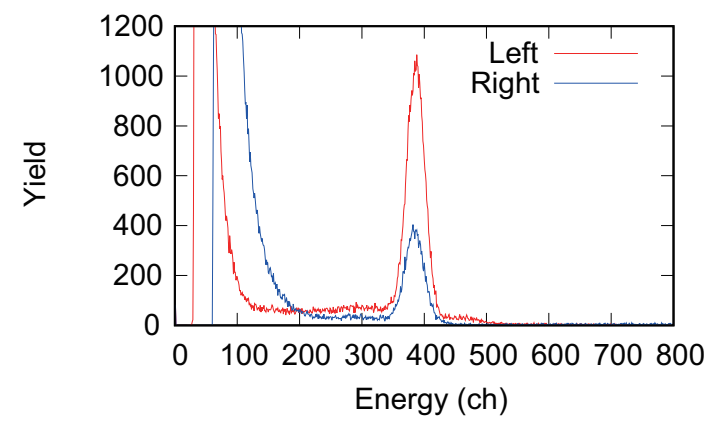

(b)

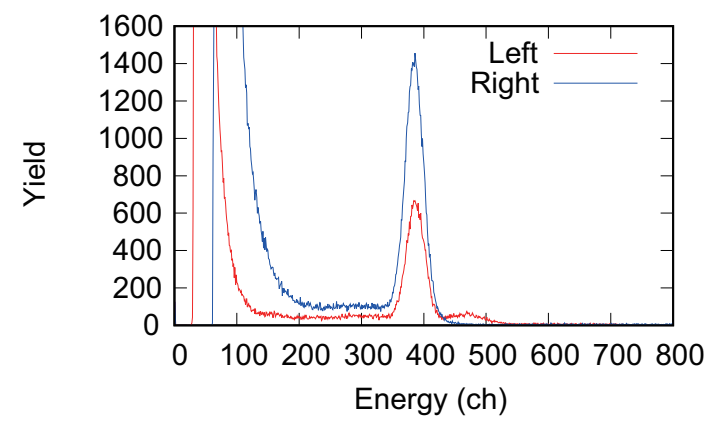

Figure 6: Energy spectra obtained from silicon detectors at the left (red line) and the right (blue line) sides of the polarimeter. Panel (a) and (b) are the results for spin-up and spin-down, respectively.

$\left.{ }^{29} \mathrm{Si}: 4.7 \%,{ }^{30} \mathrm{Si}: 3.1 \%\right)$ in order to produce ${ }^{25} \mathrm{Al}\left(t_{1 / 2}=7.2 \mathrm{sec}\right)$ via the ${ }^{28} \mathrm{Si}(\vec{p}, \alpha)$ reaction, and ${ }^{29} \mathrm{P}$ $\left(t_{1 / 2}=4.1 \mathrm{sec}\right)$ via the ${ }^{29} \mathrm{Si}(\vec{p}, n)$ reaction. Two sets of plastic scintillation counters were placed above and below the stopper in order to count the number of the $\beta$-ray emitted from the unstable nuclei, as shown in Fig. 5(b). Figure 7 shows a typical $\beta$-ray time spectrum. Experimental data were fitted by exponential functions assuming the half-lives of ${ }^{25} \mathrm{Al}$ and ${ }^{29} \mathrm{P}$. As shown in Fig. 7 , the experimental curve is well reproduced by ${ }^{25} \mathrm{Al}$ and ${ }^{29} \mathrm{P}$ components plus a constant value. From this result, we confirmed the production of ${ }^{25} \mathrm{Al}$ and ${ }^{29} \mathrm{P}$. In the future, we will attempt to measure nuclear moments of unstable nuclei with the $\beta$-ray-detected nuclear magnetic resonance ( $\beta$-NMR) technique. The preparation of the experimental system is currently in progress.

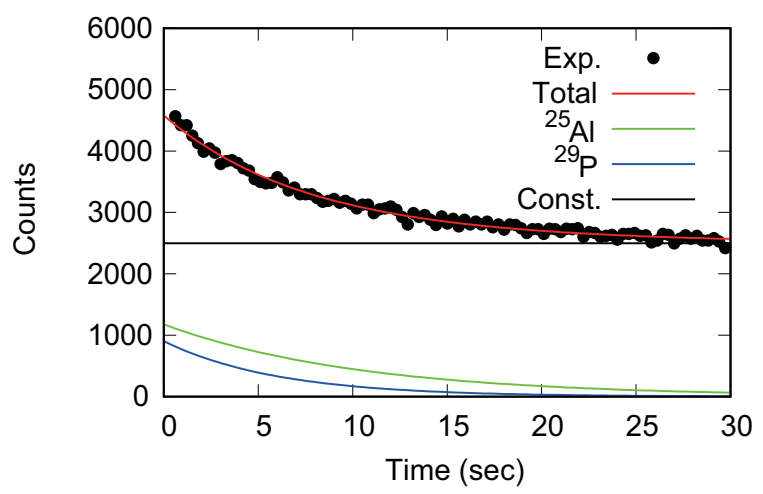

Figure 7: Typical $\beta$-ray time spectrum. Experimental data are indicated by closed circles. The red, green, blue, and black lines represent the total, ${ }^{25} \mathrm{Al},{ }^{29} \mathrm{P}$, and constant components fitted by exponential functions assuming the half-lives of these nuclei, respectively.

\section{References}

[1] Y. Tagishi et al., Nucl. Instrum. Methods Phys. 164, 411 (1979).

[2] K. Sasa et al., Nucl. Instrum. Methods Phys. Res. B 361, 124 (2015). 
[3] W. E. Lamb, Jr. and R. C. Retherford, Phys. Rev. 81, 222 (1951).

[4] W. E. Lamb, Jr., Phys. Rev. 85, 259 (1952).

[5] A. Kurashima et al., UTTAC Annual Report 1987, UTTAC-54, 14 (1988).

[6] P. W. Keaton, Jr., et al., Pys. Rev. Lett. 29, 880 (1972). 